\title{
Disposiciones de consumo informativo digital en estudiantes de periodismo*
}

\author{
Nairbis Desiree Sibrian Díaz** \\ Paulina Loreto Maureira González*** \\ Vicente Bustos**** \\ Recibido: 2020-01-30 • Enviado a pares: 2020-02-25 \\ Aprobado por pares: 2020-03-21 • Aceptado: 2020-05-22 \\ https://doi.org/10.22395/angr.v19n37a4
}

\begin{abstract}
Resumen
Desde hace algunas décadas, la convergencia de medios a través de pantallas, la digitalización de los procesos comunicativos y la emergencia de nuevas prácticas informativas reconfiguran el ejercicio y la formación periodística. El presente artículo muestra los resultados de un estudio exploratorio en torno a la manera en que los estudiantes de periodismo se informan en la actualidad y discriminan cierto contenido noticioso. El objetivo es indagar las disposiciones y preferencias de consumo informativo de estudiantes de periodismo. Para ello, se aplicó un sondeo de opinión a estudiantes de segundo año de dos universidades privadas de la Región Metropolitana de Chile como muestra intencionada. La investigación sugiere que existen habilidades digitales generalizadas en los estudiantes con énfasis distintos al informarse, es decir, esquemas de percepción que podrían generar distinciones entre preferencias informativas y verificación de la información recibida según el género y la universidad en la que estudian. Además, un ejercicio en línea realizado posteriormente arrojó que, pese a la prevalencia de habilidades digitales, sus prácticas informativas distan de una valoración noticiosa basada en métodos de comprobación.
\end{abstract}

Palabras clave: estudiante universitario; alfabetización; medios digitales; información; periodismo; educación sobre medios de comunicación.

Este artículo es resultado de la investigación del Seminario de Investigación de la carrera de Periodismo de la Facultad de Comunicaciones, Universidad del Desarrollo, Chile.

* Doctora en Sociología, Universidad Alberto Hurtado, Santiago, Chile. Académica, Universidad Santo Tomás, Santiago, Chile. Docente, Universidad del Desarrollo, Concepción, Chile. Correo electrónico: n.sibrian@udd. cl. Orcid: http://orcid.org/0000-0001-8008-5080

*** Magíster en Comunicación, Universidad Diego Portales, Chile. Profesora, Universidad del Desarrollo, Concepción, Chile. Correo electrónico: pmaureira@udd.cl Orcid: http://orcid.org/0000-0002-6448-6682

**** Licenciado en Comunicación Social, Universidad del Desarrollo, Concepción, Chile. Correo electrónico: vibustosg@udd.cl. Orcid: http://orcid.org/0000-0002-8008-7462 


\title{
Dispositions of the Informative digital Consumption in Journalism Students
}

\begin{abstract}
Since some decades ago, the convergence of media through a screen, the digitalization of communicative processes and the emergence of new informational practices reconfigure the journalism exercise and formation. This article shows the results of exploratory research around how journalism students get informed on current events and how they disfavor a certain type of news content. The main goal is to inquire about the disposition and preferences of information consumption in journalism students. For that, an opinion poll was performed to students of two private universities in the Metropolitan Region of Chile as an intentional sample. The research suggests that there are some generalized digital abilities among the students with different emphasis for informing themselves, i.e. perception schemes that might generate distinctions between informational preferences and verification of the information received according to their gender and university. Furthermore, an online exercise performed afterward, showed that despite the prevalence of digital abilities, their information practices are far from a newsworthy assessment based on checking methods.
\end{abstract}

Keywords: university student, literacy, digital media, information, journalism, digital media education.

\section{Disposições de consumo informativo digital em estudantes de Jornalismo}

\begin{abstract}
Resumo
Há algumas décadas, a convergência de mídias através de telas, a digitalização dos processos comunicativos e a emergência de novas práticas informativas reconfiguram o exercício e a formação jornalísticos. Este artigo mostra os resultados de um estudo exploratório sobre como os estudantes de Jornalismo se informam na atualidade e selecionam as notícias. O objetivo é questionar as disposições e as preferências de consumo informativo de estudantes de Jornalismo. Para isso, foi aplicada pesquisa de opinião a estudantes do segundo ano de duas universidades particulares da Região Metropolitana do Chile como amostra intencional. Esta pesquisa sugere que haja habilidades digitais generalizadas nos estudantes com ênfases diferentes ao se informarem, isto é, esquemas de percepção que poderiam gerar distinções entre preferências informativas e verificação da informação recebida segundo o gênero e a universidade em que estudam. Além disso, o exercício on-line realizado posteriormente demonstrou que, embora haja prevalência de habilidades digitais, suas práticas informativas se baseiam em uma avaliação da notícia baseada em métodos de comprovação.
\end{abstract}

Palavras-chave: estudante universitário; alfabetização; meios digitais; informação; Jornalismo; educação sobre meios de comunicação. 


\section{Introducción}

En los últimos años, el entorno digital se ha convertido en el nuevo espacio público (Echeverría, 2000; Castells, 2000) esencial no solo para comunicarse, sino también para consumir noticias e informarse (Casero-Ripollés, 2012). La mayoría de los procesos comunicativos se producen en un entorno multipantalla e hipermedial donde los recursos digitales son cotidianos (Scolari, 2016). Por otro lado, fenómenos como la infotoxicación, viralización o noticias falsas (Amorós, 2018) conllevan a que dichos recursos adquieran un rol cultural, económico y político importante, lo que plantea nuevos retos en materia social a las instituciones educativas (De Casas-Moreno, Caldeiro y Romero, 2018).

Tal panorama ofrece nuevas posibilidades de participación a los usuarios en contextos virtuales, pues pasa de ser receptores de la información a producir y compartir contenidos. Al grupo de usuarios que consume y produce información se les denomina prosumidores (Bonilla-del-Río, Diego-Mantecón, Lena-Acebo, 2018). Sin embargo, la capacidad de utilizar eficientemente los entornos digitales no se asocia únicamente al hecho de crecer en un contexto digital, pues esto no garantiza el desarrollo de competencias reflexivas respecto a los medios (Fajardo, Villalta y Salmerón, 2016).

Varias investigaciones señalan que la utilización de recursos mediáticos digitales por parte de los jóvenes no asegura un alto nivel de competencia mediática digital (Acosta-Silva, 2017; Carrasco, Sánchez y Carro, 2015). En este sentido, existen muchos mitos, realidades y preguntas en torno a los nativos digitales (Cabra y Marciales, 2009). En síntesis, tales estudios cuestionan que exista una competencia digital solo por el hecho de haber nacido en la era tecnológica (Prensky, 2001). Además, a ello se suman las brechas socioeconómicas que existen entre los grupos de personas, por lo que el desarrollo de tales competencias se complejiza aún más.

Por su parte, organismos internacionales relevan la necesidad de que las instituciones educativas promuevan la alfabetización mediática. Esto significa que las instituciones educativas deben contribuir a que la ciudadanía tenga la capacidad de acceder y de hacer uso de los medios de comunicación de forma adecuada y responsable (Comisión Europea, 2009) y evaluar la información de manera crítica (Unesco, 2008).

Ahora bien, esta educación debe ser multidimensional, es decir, debe valorar las diversas dimensiones humanas, socioeconómicas, políticas y pedagógicas (Benítez, Manrique y De Mier, 2014) para poder desarrollar competencias mediáticas y digitales en todos los ámbitos. De acuerdo con la Comisión Europea (2011), la competencia mediática supone la capacidad de percibir y analizar los mensajes de los medios de 
comunicación de un modo crítico. Por tanto, es necesario superar la perspectiva reduccionista que solo muestra un manejo instrumental de los recursos digitales (Buckingham, 2008), y comenzar a visualizar otras dimensiones e indicadores (Ferrés y Piscitelli, 2012) que también pongan foco en la influencia de los factores económicos y sociales.

Atendiendo el contexto local, algunos estudios revelan que internet constituye la vía para informarse entre los jóvenes chilenos de dieciocho a treinta y cuatro años, en detrimento de los medios tradicionales (Arriagada y Schuster, 2008). Estudios más recientes sobre el perfil de los estudiantes de periodismo en Chile (Mellado y Scherman, 2015) dan cuenta de una valoración positiva de los medios digitales predominante $(50,6 \%)$ frente a una valoración negativa de medios tradicionales, como prensa y televisión (12,5\%), en la dieta informativa de los jóvenes. No obstante, este predominio no supone competencias mediáticas digitales.

La presente investigación dialoga con estudios como el de Grijalva y Urrea (2017) cuyo objetivo es indagar los niveles de competencia digital en estudiantes universitarios de comunicación con el propósito de generar propuestas de intervención en dicho espacio. En estos espacios es urgente el uso de las herramientas digitales para innovar mecanismos de democratización y desarrollo social. Asimismo, coincide con Gutiérrez y Tyner (2012) en señalar los riesgos de limitar la educación mediática al desarrollo de la competencia digital en su dimensión instrumental.

Se reconoce la importancia de una serie de trabajos sobre tecnología y desigualdad, los cuales se preguntan si las tecnologías de información y comunicación (TIC), como internet y la World Wide Web, son beneficiosas para los estratos sociales de menores recursos y permiten superar brechas en un orden global emergente, o bien si estas, al ser introducidas de un modo asimétrico, son un motor de mayor desigualdad social (Vera, Rodríguez y Martínez, 2017), pues el complejo sistema digital reproduce inequidades especialmente en áreas como la inteligencia artificial, la computación algorítmica y los sistemas de aprendizaje (Mansell, 2017). En este sentido, estudios como el de Bauer (2017) explican que no solo se trata de la conectividad, el acceso a internet o el uso de redes sociales, sino que estas condiciones interactúan con otras fuerzas tecnológicas, económicas y políticas para configurar la situación desigual.

En el caso de Chile, investigaciones recientes señalan que el grupo de usuarios de TIC presenta una dispersión de ganancias más amplia que la del grupo de no usuarios. Por otro lado, los factores más importantes que facilitan o inhiben el acceso a internet son los ingresos, la educación, el área de residencia y el género (Flores, 2003).

La presente investigación vincula ingresos, educación y ubicación geográfica al momento de diseñar el estudio. Situado en la realidad chilena y en el contexto de 
dos universidades privadas, pero de perfiles socioeconómicos distintos, este estudio se pregunta: ¿Cómo se informan los estudiantes de periodismo en la actualidad? ¿Cuáles son sus tendencias de consumo informativo en el contexto digital? ¿Existen diferencias en cuanto a las preferencias informativas de acuerdo con la universidad en la que estudian? ¿Cuentan con herramientas de verificación de la información? Con el propósito de responder estas interrogantes, se han explorado las disposiciones informativas de jóvenes chilenos que estudian el segundo año de periodismo de dos casas de estudios de la Región Metropolitana, como una primera aproximación. Se espera, en un futuro, poder ampliar esta investigación a más universidades chilenas.

Teóricamente, se considera útil la perspectiva del sociólogo francés Pierre Bourdieu en tanto se pretenden vincular las disposiciones informativas y de valoración noticiosa con aspectos provenientes de las trayectorias educativas de los estudiantes. A su vez, esta perspectiva se combina con nuevas aproximaciones al contexto digital provenientes de corrientes que mezclan la tradición sociológica clásica con nuevos conceptos que emanan de las transformaciones digitales. A continuación, se plantean los aspectos más relevantes de esta perspectiva con el propósito de explorar las disposiciones informativas de jóvenes estudiantes de periodismo de segundo año de dos casas de estudios en Chile.

\section{Metodología}

La investigación se basa en un sondeo de opinión exploratorio y no probabilístico (Anguita et. al., 2003; Hernández, Fernández y Baptista, 2006). Cuenta con un muestreo intencional por conveniencia a estudiantes de segundo año de dos universidades chilenas con el fin de abrir la problemática planteada. La tasa de respuestas fue de un $90 \%$. Se encuestaron ciento cuarenta y seis estudiantes: $50 \%$ hombres y $50 \%$ mujeres con edades comprendidas entre dieciocho y veinticuatro años.

El cuestionario se diseñó y aplicó en formato digital. Contó con veintisiete preguntas, aunque cabe señalar que, por ser considerado exploratorio, muchas de ellas no fueron importantes para el análisis. A su vez, se pensó en una nueva aplicación, pero el contexto en Chile - las protestas ciudadanas a partir del 18 de octubre de 2019 por cambios estructurales y la crisis de la COVID-19 - no lo ha hecho posible. Por ello, se decidió publicar solo algunos datos que se consideran relevantes.

Se mencionan a las universidades como UA y UB respectivamente para resguardar la identidad de los participantes. El criterio de selección de las casas de estudio radica en que, si bien ambas son establecimientos educativos privados, estas tienen diferencias socioeconómicas marcadas. La primera (UA) es considerada de estrato social alto y la otra (UB) de estrato social medio, según sus respectivos aranceles anuales. Los aranceles de la primera son de más o menos tres millones de pesos 
chilenos y de la segunda son más o menos seis millones de pesos chilenos, de acuerdo con datos del portal de información universitaria del Ministerio de Educación de Chile (2019). Además, la primera se encuentra ubicada en el oriente de Santiago que tiene las comunas con menor índice de pobreza según la encuesta Casen (Ministerio de Desarrollo Social, 2018), mientras que la segunda se encuentra en el sur que tiene comunas con ingresos medios según la misma encuesta. La idea es poder ampliar esta encuesta a otras universidades públicas y privadas de Chile a futuro para poder establecer resultados más contundentes. No obstante, esta exploración inicial puede aportar luces sobre el panorama general.

Los criterios de selección de los estudiantes fueron i) que estudiaran periodismo, ii) que estuvieran en el segundo año de la carrera y iii) que pertenecieran a alguna de las dos universidades seleccionadas de la Región Metropolitana de Chile. Solo se tuvieron en cuenta los estudiantes que cursaban segundo año de periodismo durante el primer semestre de 2019, porque se considera que en ese nivel ya se manejan nociones relacionadas con procesos de indagación y verificación noticiosa según la malla académica de cada universidad. En la malla académica de ambas se señalan como asignaturas vistas para entonces: Análisis Crítico de Medios (en el caso de la UA), el Taller de Búsqueda de Información y el Taller de Actualidad (en el caso de la UB). Cabe destacar que los programas de estas asignaturas, desde hace un par de años, han comenzado a integrar, ejercicios de chequeo de noticias digitales (fact checkin) que comprenden:

- Verificación del autor (si es periodista o no).

- Verificación de la fuente (si es una fuente oficial, si hay canales directos con ella y posibilidades de ubicarla).

- Precisión de la información (fiabilidad).

Por ello, a la encuesta se le suma una segunda fase de la investigación en la que se pretende conocer la apropiación que tienen los estudiantes de periodismo sobre técnicas de verificación noticiosa. En este aspecto, la investigación no solo se dirige a los estudiantes de segundo año, sino de la carrera completa. En este sentido, se realiza un ejercicio en línea a ciento sesenta y dos estudiantes de un total de doscientos setenta y un estudiantes inscritos de la carrera de periodismo de una de las universidades exploradas (UA). Este grupo está compuesto por treinta y nueve alumnos de primer año que corresponden al $25 \%$, cuarenta y dos alumnos de segundo año que corresponden al $27 \%$, veintinueve alumnos de tercer año que corresponden al $18 \%$, veintinueve alumnos de cuarto año que corresponden al $18 \%$ y veintitrés alumnos de quinto año que corresponden al $12 \%$ del total de la muestra. Se escoge esta universidad para realizar esta segunda parte porque esta muestra se manifiesta 
mucho más familiarizada con los medios digitales en el primer instrumento aplicado y porque se quiso explorar sus capacidades de verificación.

El ejercicio se llevó a cabo a través de una plataforma digital que contaba con tres noticias falsas y una verdadera con el fin de determinar si los estudiantes cuentan con competencias mediáticas de valoración noticiosa que les permitan reconocer una noticia falsa. Los estudiantes debían indicar, sin recurrir a la apertura de otra página web, si consideraban que las noticias que leían eran verdaderas o falsas, si les parecían fiables y describir las acciones que realizarían para constatar su veracidad. El ejercicio buscaba indagar los pasos que seguían los estudiantes para verificar noticias, pues esto es algo que se les ha enseñado en las asignaturas Análisis Crítico de Medios en el caso de la UA y en Taller de Actualidad en la UB, como se ha señalado. Las noticias que se utilizaron fueron adecuadas al contexto de ese momento (primera semana de octubre de $2019^{1}$ ) para generar mayor verosimilitud.

Para el análisis se hicieron descripciones y un mapa perceptual de un análisis de correspondencias múltiples (ACM) con el fin de observar algunas relaciones para considerarlas en la ampliación de este estudio.

\section{Habitus digital y capital tecnológico}

El habitus digital tiene su origen en la sociología de Pierre Bourdieu. Este propone una aproximación a las nuevas identidades estudiantiles que surgen a partir de la incorporación de las tecnologías de la información y comunicación (Casillas y Ramírez, 2018). Estas identidades no solo comprenden hábitos, sino que se transforman en disposiciones o principios incorporados.

Juliette Grange (2011) ofrece una muy interesante genealogía del concepto de habitus, pues explica que Bourdieu lo retoma tanto de la filosofía como de la sociología clásica. En la filosofía de Aristóteles, habitus se utilizaba para hablar de hexis que significaba disposición, lo cual se podía entender, a su vez, como una forma de capital. Posteriormente, Bourdieu (1987) propone una serie de dimensiones asociadas al habitus a partir de los términos eîdos (sistema de esquemas lógicos o de estructuras cognitivas que organizan las visiones del mundo), ethos (conjunto de disposiciones morales), aísthesis (el gusto o la disposición estética) y hexis (maneras de ser del cuerpo).

No obstante, el mismo Bourdieu señala que tales dimensiones son solo analíticas, ya que el habitus se expresa de manera indiferenciada o entremezclada (Casillas y Ramírez, 2018) tanto en el plano cognitivo (conocimiento, percepción) como en el axiológico (apreciación, evaluación) y el práctico (gesto, postura). Por tanto, se puede ver reflejado en múltiples acciones cotidianas.

\footnotetext{
Antes del estallido social ocurrido a partir del 18 de octubre de 2019 en Chile.
} 
Siguiendo estos postulados, Julien (2015) hace un análisis detallado de las implicaciones teóricas del uso de conceptos de Bourdieu en la interacción en línea. Al considerar la esfera virtual como un campo en sí mismo, que exige el desarrollo de una serie de habilidades (mediáticas y digitales) y conlleva a una inversión por parte de los usuarios en materia de tiempo y recursos, el autor sostiene que existe un habitus en internet que los usuarios deben conocer si quieren moverse socialmente de forma adecuada.

Posteriormente, en el marco de la cultura digital, Casillas y Ramírez (2018) proponen que el habitus se puede ver reflejado en diversas dimensiones, las cuales van desde las disposiciones morales hasta las posturas corporales. En este estudio se hará énfasis en los sistemas y estructuras cognitivas que organizan visiones de mundo (ềdos) y que incluyen las habilidades para interactuar en ambientes virtuales a través del uso de aparatos electrónicos y de información digital.

La posibilidad de que el habitus pueda ayudar a explicar cierto comportamiento dentro del mundo de las nuevas tecnologías educativas en la vida cotidiana (Cerón, 2016) y de los modos en que se reconfiguran las prácticas informativas en las nuevas generaciones, conduce a pensar que dicho habitus depende de un capital tecnológico (Casillas y Ramírez, 2018) que incluye competencias en torno al uso de dispositivos digitales, el consumo de los contenidos y la valoración de los mismos en torno a su credibilidad o autoridad.

\section{Jóvenes y consumo informativo}

El sector juvenil usa las redes en mucha más proporción que los adultos. Esta tendencia se extiende con independencia de la renta de las familias con acceso a internet (Bernal y Angulo, 2013). Por tanto, es posible inferir que los "nativos digitales" (Prensky, 2005) tienen más conocimientos tecnológicos que los "inmigrantes digitales". No obstante, esto no implica un mayor grado de desarrollo de la dimensión del lenguaje o la producción de contenidos.

De acuerdo con investigaciones recientes (Taddeo, 2019), los jóvenes usan a menudo sistemas de índice de audiencia de las plataformas digitales o usan el "bocaoreja" en línea en las redes sociales y en los grupos de WhatsApp como métodos para subvertir los sistemas tradicionales de mediación cultural y para elegir artículos, libros y contenidos fuera de los circuitos "estándares" de los medios de comunicación de masas y de las agencias tradicionales de mediación cultural.

Un estudio llevado a cabo en contexto italiano en más de nueve mil quinientos estudiantes (Buffardi y Taddeo, 2017) revela que los jóvenes con alto rendimiento escolar están más interesados en ampliar sus capacidades digitales, mientras que los estudiantes con notas más bajas tienden a preocuparse menos por desarrollar 
competencias digitales y a priorizar actividades de entretenimiento, comunicación y diversión digital.

Los retos propiciados por las diversas formas de interacción social, incluyendo las narrativas transmediales (Guerrero-Picó y Scolari, 2016), requieren que las instituciones educativas comprendan esta realidad. De esta manera, podrían desarrollar de una manera más adecuada los procesos de formación académica de acuerdo con los adelantos emergentes provistos por la era de la cultura digital.

\section{Valoración noticiosa y noticias falsas}

Los criterios de valoración noticiosa están relacionados con la alfabetización mediática (media literacy), la cual se define como la capacidad de acceder, analizar, evaluar y crear contenidos mediáticos en una variedad de formas. Concretamente, está vinculada con la formación. Su propósito es ayudar a que las personas tengan criterios y argumentos para tomar mejores decisiones (Lucas, Couto y Perea, 2018), lo cual incluye habilidades relacionadas tanto con la recepción como con la producción (Aguaded, Marín y Díaz, 2015). Dentro de esta, está la competencia mediática (media competence), la cual consiste en consultar, comprender y evaluar críticamente, así como crear contenido en los medios (Parlamento Europeo, 2007).

En este sentido, la alfabetización mediática se amplía hacia el terreno de lo digital (digital literacy) y recoge el uso de las tecnologías de la información y la comunicación (digital competence), ahora con la capacidad de localizar, organizar, comprender, evaluar y analizar información con el uso tecnología digital. Esta competencia incide en aspectos tales como la reflexión crítica de los usuarios (Tíscar, 2009).

En la actualidad, la mayoría de los procesos comunicativos actuales son multipantalla e hipermediales (Scolari, 2016), mientras que los fenómenos como la infodemia, viralización o noticias falsas (Amorós, 2018) adquieren un rol cultural, económico y político importante. Este hecho plantea nuevos retos en materia social y educativa (De Casas-Moreno, Caldeiro y Romero, 2018) a nivel global.

Un estudio sobre el impacto de las noticias falsas en España afirma que el 86 \% de los españoles tiene dificultades para distinguir las noticias verdaderas de las que no lo son y un 90 \% ha compartido estas últimas por diversión (García y Évole, 2018). En Chile, el panorama parece ser más alentador. Según un estudio realizado por investigadores de la Universidad Católica, más del $75 \%$ de usuarios de internet a nivel nacional está al tanto de al menos cuatro afirmaciones falsas en la web, especialmente las relacionadas con rumores. Por otro lado, el $24 \%$ de los encuestados considera que tales afirmaciones son "muy" o "extremadamente creíbles" (Valenzuela et al., 2019). 
Por tanto, estar siempre en línea supone recibir centenares de información cada día sin la capacidad de dedicar el tiempo suficiente para analizar si son fiables, lo que puede significar desinformación (Lazer et al., 2018) y hasta infotoxicación (Gómez, 2016) a partir de la circulación de numerosas noticias falsas (Herrero et al., 2019). De ahí la importancia de generar mecanismos de verificación de la información que puedan contribuir a que las personas tomen decisiones mucho más conscientes.

Para arrojar luz sobre la evaluación de la fiabilidad de la información, aparece el modelo Craap de Blakeslee (2004), el cual tiene el propósito de facilitar la valoración de la información. Este surge como una recomendación que hay que tener en cuenta para enjuiciar la información. No obstante, en la actualidad se ha consolidado como una línea de investigación. Dicho modelo incluye los siguientes aspectos: i) actualidad (puntualidad de la información); ii) relevancia (la importancia de la información para sus necesidades); iii) autoría (la fuente de la información); iv) precisión (fiabilidad del contenido) y v) propósito (motivo por el que existe la información).

Varios de estos aspectos se han venido incluyendo en las mallas de las carreras de periodismo, específicamente en asignaturas prácticas, como los talleres que involucran los medios y la búsqueda de información y las asignaturas analíticas que suponen la reflexión sobre el rol de la información en la actualidad.

\section{Resultados}

Los resultados están divididos en dos apartados. El primero da cuenta de los datos obtenidos en la aplicación del cuestionario a los estudiantes de periodismo de dos universidades de estratos socioeconómicos distintos (UA y UB). El segundo apartado muestra en detalle la indagación con respecto a las competencias de valoración noticiosa de estudiantes de periodismo de todos los niveles en una sola universidad (UA).

\section{El habitus digital en dos universidades}

Uno de los primeros resultados que arrojó la encuesta fue la dimensión común a los estudiantes de ambas universidades en el uso del mundo digital-virtual para informarse, donde el 90 \% utilizan o las redes sociales o los medios digitales para tal efecto. Sin embargo, como se aprecia en la tabla 1, existe una diferencia entre ambas universidades la cual advierte que la mayoría de los estudiantes de la UB (de estrato socioeconómico medio) prefiere informarse a través de redes sociales. Por otro lado, los estudiantes de la UA (de estrato socioeconómico alto) se inclinan más hacia medios digitales. La tendencia exploratoria de este estudio confirma que los medios tradicionales como la prensa impresa, la radio y la televisión ya no son las principales opciones de los jóvenes para informarse a diario, incluso si estudian periodismo. 
Tabla 1. ¿Qué medio utilizas diariamente para informarte sobre lo que sucede en el país?

\begin{tabular}{lccc}
\hline \multicolumn{1}{c}{ Medios } & UA & UB & Total \\
\hline Redes sociales & $41,7 \%$ & $76,0 \%$ & $47,6 \%$ \\
\hline Medios digitales & $54,2 \%$ & $12,0 \%$ & $46,9 \%$ \\
\hline Medios impresos & $1,7 \%$ & $4,0 \%$ & $2,1 \%$ \\
\hline Radio y TV & $2,5 \%$ & $8,0 \%$ & $3,4 \%$ \\
\hline Total & $100,0 \%$ & $100,0 \%$ & $100,0 \%$ \\
\hline
\end{tabular}

Fuente: elaboración propia.

Instagram resulta ser la red social preferida para la mayoría de los estudiantes (54 \%), seguido de Twitter (34 \%) y Facebook (12 \%). En la figura 1, elaborada a partir de un análisis de correspondencias múltiples, se advierten algunas diferencias entre ambas universidades, donde la disparidad de capital económico parece marcar algunas distinciones en las preocupaciones temáticas e, incluso, en la posibilidad de informarse. Por ejemplo, si bien la mayoría usa más las redes sociales, los estudiantes de estratos económicos altos (UA) se hallan más cerca de los medios impresos y digitales, quizás por el hecho de que en sus hogares se leen estos o están suscritos a sus versiones en internet. Por el contrario, los estudiantes de estratos económicos bajos (UB) tienen mayor cercanía con las redes sociales y con la conversación como medio de información.

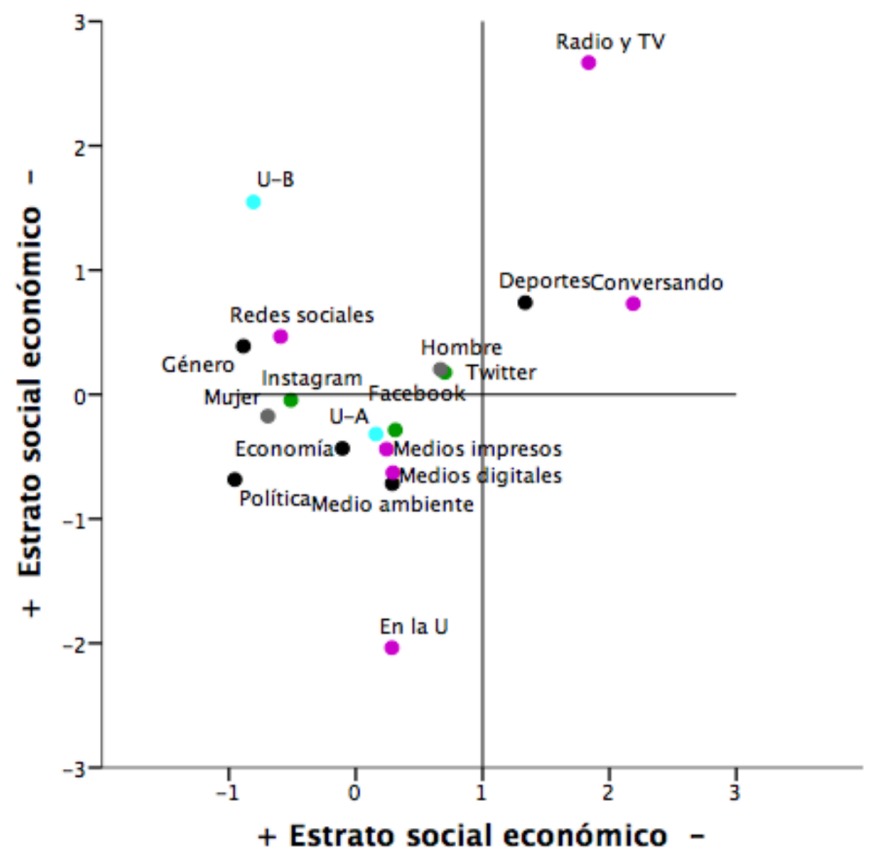

Figura 1. Análisis de correspondencias múltiples

Fuente: elaboración propia. 
Otro problema que se muestra en esta figura es la incidencia de temas preferidos por los estudiantes de ambas universidades: los estudiantes de la UA son más cercanos a temas políticos, económicos y medioambientales, mientras que los estudiantes de la UB son más cercanos a temas de género y deportes.

Por último, se puede hacer una diferencia por género: los hombres, en cuanto al uso de redes sociales, son más cercanos a Twitter, mientras que las mujeres son más cercanas a Instagram. Asimismo, los hombres tienen una mayor relación con temas deportivos, mientras que las mujeres con problemáticas de género.

Un aspecto interesante que surge de esta encuesta a los estudiantes de periodismo en ambas universidades es que, a pesar de que la mayoría utiliza las redes sociales para informarse, muy pocos las usan para compartir noticias o información relevante del acontecer cotidiano local o internacional. Solo el $13 \%$ entre ambas universidades señala que comparte este tipo de información en sus redes sociales, pues la mayoría las utilizan con fines personales y cotidianos. Sin embargo, también señalan que rara vez lo pueden hacer (66 \%) si la noticia les interesa y sienten que a través de la misma pueden decir algo que los represente (75\%). Esto quiere decir que, de los estudiantes de periodismo de ambas universidades, solo una pequeña porción comparte noticias con el fin de informar, ya sea bajo la excusa de ver la reacción de los demás contactos o porque lo considera, a priori, una forma de interacción con la comunidad y parte de su oficio (15\%).

Otro hallazgo interesante es que solo el $56 \%$ de estudiantes de periodismo que señalaron que podían compartir alguna noticia en sus redes sociales dijeron que trataban de verificar el origen de la noticia, lo que conlleva un problema para la formación de nuevos periodistas. Por otro lado, apenas el 11 \% manifestó que verificaban quién había escrito la noticia antes de compartirla, lo cual es fundamental en la formación periodística.

Con estos resultados se vislumbra la siguiente discusión en torno a la formación de un habitus digital informativo con el fin de abrir interrogantes sobre la incidencia del capital cultural en el desarrollo de competencias comunicativas de valoración noticiosa.

\section{Competencias de valoración noticiosa en una universidad}

Tras la revisión de los resultados arrojados por el cuestionario, la investigación se plantea un ejercicio exploratorio de la valoración noticiosa de los estudiantes de una de las universidades pesquisadas. Se consideró a la UA para este efecto por temas de logística interna y acceso. De una población total de doscientos setenta y un estudiantes matriculados en la carrera en el año 2019, ciento sesenta y dos estudiantes se enfrentaron a un desafío de valoración noticiosa para poder observar 
su capacidad para discriminar entre noticias falsas y verdaderas, en relación con la dimensión de recepción del concepto de competencias mediáticas (Ferrés, 2007). El ejercicio consistió en responder cuatro preguntas de actualidad en las que se debía distinguir cuáles eran falsas (tabla 2).

Tabla 2. ¿Puedes identificar si los siguientes titulares son noticias verdaderas o falsas?

\begin{tabular}{lccc}
\hline \multicolumn{1}{c}{ Titular } & Verdadera & Falsa & Total \\
\hline $\begin{array}{l}\text { 1. Trump logró la mayor audiencia televisiva de una investidura } \\
\text { presidencial en Estados Unidos. }\end{array}$ & $78,3 \%$ & $21,6 \%$ & $100,0 \%$ \\
\hline $\begin{array}{l}\text { 2. Los rusos manipularon las elecciones presidenciales de Estados } \\
\text { Unidos. }\end{array}$ & $62,3 \%$ & $37,3 \%$ & $100,0 \%$ \\
\hline $\begin{array}{l}\text { 3. Greta Thunberg ha sido financiada por grandes empresarios } \\
\text { como George Soros. }\end{array}$ & $47,5 \%$ & $52,4 \%$ & $100,0 \%$ \\
\hline \begin{tabular}{l} 
4. Delta Airline está negociando la compra del 20\% de Latam. \\
\hline
\end{tabular} & $56,1 \%$ & $43,8 \%$ & $100,0 \%$ \\
\hline
\end{tabular}

Fuente: elaboración propia.

Ante la primera interrogante, la mayoría de estudiantes consultados respondió que la noticia era verdadera. Sin embargo, esta noticia es falsa. A pesar de que Donald Trump señaló en febrero de 2017 que logró la mayor audiencia de la historia de una investidura presidencial (45,6 millones de televidentes), la realidad es que la de Barack Obama en 2010 tuvo una mayor audiencia (seguida por 48 millones de personas). Incluso Trump fue desmentido por varios medios de comunicación después de aseverar tal afirmación.

La segunda pregunta también es falsa. Pero, nuevamente, la mayor parte de los encuestados respondió que la noticia era verdadera, pues este tipo de noticias responden a ciertas ideas perceptivas (como teorías conspirativas) que suelen tener bastante audiencia y réplica en las redes sociales, dada la personalidad de Trump. Con la tercera pregunta sucede algo similar, pero de forma inversa. La mitad considera que la premisa es verdadera, mientras que la otra mitad la considera falsa, pues muchas personas le asignan cierto rol moral a una activista como Greta Thunberg que implica que ella no podría ser financiada por grandes empresarios si tiene cierta lucha ética por el medio ambiente. Pero, a pesar de que un poco más de la mitad se inclina a afirmar que esta noticia es falsa, la otra mitad de los estudiantes cree que podría ser cierto, pues resulta verosímil. En la cuarta y última pregunta, un poco más de la mitad cree que la noticia es verdadera. Efectivamente, Delta Airlines negoció en un momento la compra del $20 \%$ de Latam y, aunque esta podría ser la pregunta más difícil, nuevamente se juega con la idea de que sea verdadera a causa de la coyuntura y apertura económica de Chile. 
Con este simple ejercicio se percibe un problema significativo en relación con la formación de estudiantes de periodismo en la actualidad y la idea de un posible habitus digital. Este habitus se vincula al hecho de que se pueden compartir muchas noticias que se consideran verdaderas sin comprobar su veracidad, solo por quien aparece en esta. Esto quiere decir que la carga moral de ciertos personajes conlleva a valorar la noticia de forma positiva o negativa, lo que conduce a la no generación de sospechas y, por ende, a la no comprobación de sus fuentes.

Algunos de los encuestados de la UA (10 \%) cree que las noticias falsas no tienen respaldo de sus fuentes y un $10 \%$ cree que solo son títulos llamativos y noticias que combinan una historia verdadera con una falsa, lo que aleja, de cierta forma, del oficio de consulta de fuentes. A su vez, se plantea la pregunta sobre si el capital socioeconómico no garantiza, en muchos casos, la creencia o no en noticias falsas.

\section{Discusión y conclusiones}

Con respecto al objetivo principal de la investigación, el cual consistía en explorar la manera en que se informan los estudiantes de periodismo en dos universidades chilenas, los resultados de la encuesta sugieren que la gran mayoría de los estudiantes de periodismo de ambas universidades utiliza las redes sociales o los medios digitales para acceder a la información, aunque se aprecia una diferencia entre ambas universidades respecto a los medios por los cuales se informan. De acuerdo con una perspectiva bourdieusiana, en el caso de la UA (estrato social alto) la inclinación por los medios digitales podría estar influida por un capital cultural heredado conformado por el capital familiar y el capital escolar (Vera, Rodríguez y Martínez, 2017). Este capital otorga mayor legitimidad a los medios tradicionales. En este sentido, no sería extraño encontrar que en sus familias estén suscritos a algunos medios impresos o digitales. Por el contrario, en el caso de los estudiantes de la segunda universidad (UB) es probable que la relación con la información venga más por la televisión o radio. Por ende, el desapego de los medios formales es mucho más patente que en la otra universidad.

El análisis de correspondencias múltiples muestra, sin embargo, algunos desafíos comunes para el caso de ambas universidades en su proceso de formación. La diferencia socioeconómica entre ambas universidades muestra que los temas de interés tienen cierta relación con sus realidades. Esto implica un desbalance en los temas, pues se pueden crear algunas especializaciones en algunos temas y un desconocimiento total en otros, lo que también podría impactar la inserción en el ámbito laboral.

Por otro lado, este análisis muestra una desventaja de la UB con respecto a la UA porque, al interrogar por las noticias falsas en la segunda universidad, se puede notar 
un habitus digital generacional. El mapa perceptual muestra que la universidad de estrato social bajo se halla más cerca de las redes sociales, lo que puede implicar que estén más propensos a las noticias falsas. Sin embargo, en la universidad de estrato social alto no hubo respuestas tan asertivas en el ejercicio de verificación de algunas noticias falsas, lo que indica que existe un habitus digital en las nuevas generaciones propensas a la inmediatez de la noticia. Ello se ve reflejado, además, en que la mayoría de los estudiantes encuestados declaran ser más consumidores de noticias que productores o difusores, y cuando difunden una noticia no comprueban las fuentes.

El desarrollo de competencias mediáticas como parte de un habitus digital aparece, entonces, como un gran desafío común entre ambas universidades, pues pocos verifican una noticia ( $50 \%$ aproximadamente) y muy pocos constatan el autor de una nota como forma de otorgar credibilidad a un contenido. La característica de consumidores, más que de productores de información, guarda relación con el hecho de tener el hábito de comprobar fuentes. Aunque más de la mitad de los estudiantes encuestados comparte noticias ocasionalmente, estas publicaciones responden a sus intereses personales.

Tales hallazgos pueden vincularse con lo que dicen otras investigaciones sobre jóvenes y uso de redes sociales (Bernal y Angulo, 2013). Estas investigaciones señalan que las motivaciones afectivas y sociales predominan en detrimento de las informativas, lo que conduce a que los jóvenes consuman o difundan cierto tipo de información en sus redes sociales.

Otro resultado que se muestra es un cambio en los asuntos declarados prioritarios en las dietas informativas de los estudiantes de periodismo. Política y economía, por ejemplo, pierden terreno ante temas como género y medioambiente. Por otro lado, se podría decir que los deportes son un tema más o menos transversal a ambas universidades, el cual no pierde vigencia hasta el día de hoy. Todo ello plantea un escenario de cambio en los intereses de los periodistas del mañana. Asimismo, estos cambios muestran la manera en que el proceso de formación de los estudiantes de periodismo hace converger temas nuevos con otros campos necesarios, tales como la economía y la política.

De esta forma, la categoría de habitus digital puede aportar luces con respecto a las prácticas asociadas a los usos y las preferencias de la información en jóvenes estudiantes de periodismo en tiempos actuales desde una dimensión crítica y reflexiva. Con estos resultados es imperativo comenzar a revisar la manera en que las escuelas de periodismo no solo enfrentan las modalidades de acceso al entorno digital, sino las prácticas cotidianas que conlleven hacia un discernimiento informativo responsable y competencias de valoración noticiosa eficaces. 


\section{Referencias}

Acosta-Silva, D. (2017). Tras las competencias de los nativos digitales: avances de una metasíntesis. Revista Latinoamericana de Ciencias Sociales, Niñez y Juventud, 15(1), 471-489.

Aguaded, I., Marín, I. y Díaz, E. (2015). Media literacy between primary and secondary students in Andalusia (Spain). Revista Iberoamericana de Educación a Distancia, 18(2), 275-298.

Amorós, M. (2018). Fake News. la verdad de las noticias falsas. Plataforma Editorial.

Anguita, J., Labrador, J., Campos, J., Casas, J., Repullo, J. y Donado, J. (2003). La encuesta como técnica de investigación. Elaboración de cuestionarios y tratamiento estadístico de los datos (I). Atención primaria, 31(8), 527-538.

Arriagada, A. y Schuster, M. (2008). Consumo de medios y participación ciudadana de los jóvenes chilenos. Cuadernos de Información, 22(1), 34-41.

Bauer, J. (2017). The Internet and income inequality: Socio-economic challenges in a hyperconnected society. Telecommunications Policy, 42(4), 333-343.

Benítez, M., Manrique, S. y De Mier, V. (2014). Las dimensiones sociales, cognitivas y lingüísticas del proceso de alfabetización en jóvenes y adultos. Revista Interamericana de Educación de Adultos, 37(1), 89-106.

Bernal, C. y Angulo, F. (2013). Interacciones de los jóvenes andaluces en las redes sociales. Comunicar, 40, 25-30. http://dx.doi.org/10.3916/C40-2013-02-02

Blakeslee, S. (2004). The CRAAP Test. En Loex Quarterly, 31(3), 6-7.

Bonilla-del-Río, M., Diego-Mantecón, J. y Lena-Acebo. F. (2018). Estudiantes Universitarios: prosumidores de recursos digitales y mediáticos en la era de Internet. Aula Abierta, 47(3), 319-326.

Buffardi, A. y Taddeo, G. (2017). The Web 2.0 Skills of Italian Students: An Empirical Study in Southern Italy. Italian Journal of Sociology of Education, 9(1), 45-76.

Bourdieu, P. (1987). Los tres estados del capital cultural. Sociológica, 2(5), 11 17.

Buckingham, D. (2008). Más allá de la tecnología. Aprendizaje infantil en la era de la cultura digital. Ediciones Manantial.

Cabra, F. y Marciales, G. (2009). Mitos, realidades y preguntas de investigación sobre los nativos digitales: una revisión. Universitas Phychologica, 8(2), 323-338.

Casillas, M. y Ramírez, A. (2018). El habitus digital: una propuesta para su observación. En R. Castro y H. Suárez (coords.). Pierre Bourdieu en la sociología latinoamericana. El uso de campo y habitus en la investigación (pp. 207-225). Universidad Autónoma de México.

Carrasco, M., Sánchez, C. y Carro, A. (2015). Las competencias digitales en estudiantes del posgrado en educación. Revista Lasallista de Investigación, 12(2), 10-18.

Casero-Ripollés, A. (2012). Más allá de los diarios: el consumo de noticias de los jóvenes en la era digital. Comunicar, 39(2), 151-158. https://doi.org/10.3916/C39-2012-03-05 
Castells, M. (2000). La era de la información: economía sociedad y cultura. Vol. 1: La sociedad red. Alianza editorial.

Cerón, A. (2016). El uso de conceptos teóricos de Pierre Bourdieu en la investigación educativa en México. Revista de Ciencias Sociales, 71, 192-208.

Comisión Europea. (2009, 22 de agosto). Commission recommendation of 20 August 21011 on media literacy in the digital environment for a more competitive audiovisual and content industry and an inclusive knowledge society. Official Journal of the European Union, 227(9). https:// goo.gl/ofZwbz

De Casas-Moreno, P., Caldeiro, M. y Romero, L. (2018). La televisión como espejo de la realidad del "bullying". Percepciones de los adolescentes sobre el programa "Proyecto Bullying". Aula Abierta, 47(2), 193-202.

Echeverría, J. (2000). Un mundo virtual. Gedisa.

Fajardo, I., Villalta, E. y Salmerón, L. (2016). ¿Son realmente tan buenos los nativos digitales? Relación entre las habilidades digitales y la lectura digital. Anales de Psicología, 32(1), 89-97. http://dx.doi.org/10.6018/analesps.32.1.185571

Ferrés, J. (2007). La competencia en comunicación audiovisual: Dimensiones e indicadores. Comunicar, 15(29), 100-107. https://doi.org/10.3916/C29-2007-14

Ferrés, J. y Piscitelli, A. (2012). La competencia mediática: propuesta articulada de dimensiones e indicadores. Comunicar, 19(38), 75-82.

Flores, C. (2003). Measuring the relationship between ICT use and income inequality in Chile. Working Paper, 26. https://utip.lbj.utexas.edu/papers/utip_26.pdf

García, M. y Évole, J. (2018). Fake News: La verdad de las noticias falsas. Plataforma.

Grange, J. (201 1). El Habitus, de la Filosofía a la sociología, ida y vuelta. En M. Lescourret (coord.) Pierre Bourdieu. Un filósofo de la sociología (pp. 125-139). Nueva Visión.

Gómez, B. (2016): El consumidor ante la infoxicación en el discurso periodístico. Estudios sobre el Mensaje Periodístico, 22(1), 313-327.

Grijalva, A. y Urrea, M. L. (2017). Evaluación de la competencia digital en estudiantes de comunicación. El caso de una universidad mexicana. Edmetic, 6(2), 276-301. https://helvia. uco.es/bitstream/handle/10396/15411/Edmetic_vol_6_n_2_16.pdf?sequence =1 1 EisAllowed =y

Guerrero, M. y Scolari, C. (2016). Narrativas transmedia y contenidos generados por los usuarios: El caso de los crossovers. Cuadernos.info, (38), 183-200. http://dx.doi.org/10.7764/cdi.38.760

Gutiérrez, A. y Tyner, K. (2012). Media Education, Media Literacy and Digital Competence. Comunicar, 19(38), 31-39. https://doi.org/10.3916/C38-2012-02-03

Herrero, P., Conde, J., Tapia, A. y Varona, D. (2019). The credibility of online news: an evaluation of the information by university students. Culture and Education, 31(2), 407-435.

Hernández, R., Fernández, C. y Baptista, P. (2006). Metodología de la Investigación (4ª edición). McGraw-Hill. 
Julien, C. (2015). Bourdieu, Social Capital and Online Interaction. Sociology, 49(2), 356-373.

Lazer, D., Baum, M., Benkler, Y., Berinsky, A., Greenhill, K., Menczer, F. y Schudson, M. (2018). The science of fake news. Science, 359(6380), 1094-1096.

Lucas, C., Couto, M. y Perea, M. (2018). Digital literacy and education report by country. European Literacy Network. https://www.eln.eu/fotos/editor2/imagens/spain_report.pdf

Mansell, R. (2017). The mediation of hope: communication technologies and inequality in perspective. International Journal of Communication, 11, 4285-4304.

Mellado, C., y Scherman, A. (2015). Estudiantes de periodismo en Chile: Percepción sobre la profesión, su futuro laboral y el desempeño de los medios. Universidad Católica de Valparaíso.

Ministerio de Desarrollo Social. (2018). Encuesta Casen. http://observatorio.ministeriodesarrollosocial. gob.cl/casen-multidimensional/casen/casen_2017.php

Ministerio de Educación, Subsecretaría de Educación superior (2019, 12 de diciembre) Portal mifuturo.cl actualiza datos de ingreso y empleabilidad de las carreras. https://educacionsuperior. mineduc.cl/2019/12/12/portal-mifuturo-cl-actualiza-datos-de-ingreso-y0-empleabilidad-delas-carreras/

Parlamento Europeo. (2007). Study on the current trends and approaches to media literacy in Europe. https://ec.europa.eu/assets/eac/culture/library/studies/literacy-trends-report_en.pdf

Prensky, M. (2001). Nativos e inmigrantes digitales. Distribuidora SEK. http://goo.gl/8DA7t

Prensky, M. (2005). Engage me or enrage me. What today's learners demand. Educause Review, 40(5), 60-65. http://bit.ly/21S6950

Scolari, C. (2016). Alfabetismo transmedia: estrategias de aprendizaje formal y competencias mediáticas en la nueva ecología de la comunicación. Telos: revista de pensamiento sobre Comunicación, Tecnología y Sociedad, (103), 13-23.

Taddeo, G. (2019). El uso de lo digital para desarrollar estrategias de aprendizaje informal: Estudios y herramientas. En I. Aguaded, A. Vizcaíno-Verdú y Y. Sndoval-Romero (eds.). Competencia mediática y digital: del acceso al empoderamiento (pp. 39-55). Ediciones Grupo Comunicar.

Tíscar, L. (2009). La competencia digital en el área de lengua. Octaedro.

Unesco. (2008, 16 de junio). Teacher training curricula for media and information literacy. Report of the International Expert Group Meeting. International Unesco. http://www.unesco.org/new/fileadmin/ MULTIMEDIA/HQ/CI/CI/pdf/teacher_training_curricula_mil_meeting_june_2008_report_en.pdf

Valenzuela, S., Halpern, D., Katz, J. y Miranda, J. (2019). The Paradox of Participation Versus Misinformation: Social Media, Political Engagement, and the Spread of Misinformation. Digital Journalism, 7(6), 802-823. https://doi.org/10.1080/21670811.2019.1623701

Vera, J., Rodríguez, C. y Martínez, E. (2017). Capital cultural y competencias digitales en estudiantes universitarios. Ventana Informática, (36), 99-116. 